\title{
Dual Pathway Inhibition with Low-Dose Direct Factor Xa Inhibition after Acute Coronary Syndromes-Why Is It Not Used in Clinical Practice?
}

\author{
Uwe Zeymer ${ }^{1}$ Benedikt Schrage ${ }^{2}$ Dirk Westermann² \\ ${ }^{1}$ Klinikum Ludwigshafen and Institut für Herzinfarktforschung \\ Ludwigshafen, Ludwigshafen, Germany \\ 2 Klinik für Allgemeine und Interventionelle Kardiologie, Universitäres \\ Herzzentrum Hamburg, Hamburg, Germany \\ Address for correspondence Uwe Zeymer, MD, Klinikum \\ Ludwigshafen and Institut für Herzinfarktforschung, Bremserstrasse \\ 79, D-67063 Ludwigshafen/Rhein, Germany \\ (e-mail: uwe.zeymer@t-online.de).
}

Thromb Haemost 2018;118:1528-1534.

\begin{abstract}
Keywords

- acute myocardial infarction

- anti-platelet agents

- clinical trials

- oral anticoagulants
\end{abstract}

The optimal anti-thrombotic therapy for secondary prevention after an acute coronary syndrome is still a matter of debate. While current guidelines recommend dual antiplatelet therapy with aspirin and a P2Y12 inhibitor over 12 months especially in patients with stent implantation, the value of prolonged anticoagulation is still controversial. In the ATLAS-TIMI 52 trial, a low-dose direct factor Xa inhibition with rivaroxaban compared with placebo reduced the combined primary endpoint of cardiovascular mortality, myocardial infraction and stroke with an increase in major bleeding complications. This article discusses the value and problems of adding low-dose rivaroxaban to anti-platelet therapy as secondary prevention measure after an acute myocardial infarction. It will describe the pros and cons of intensified anti-platelet therapy versus dual pathway inhibition and give recommendations for different patient groups in clinical practice.

\section{Introduction}

Platelet aggregation and thrombus formation are key components in the pathophysiology of acute coronary syndromes (ACSs). ${ }^{1}$ Therefore, anti-thrombotic therapy is a cornerstone of treatment in patients with ACS. ${ }^{2,3}$ Several large-scale trials have shown that platelet inhibition with aspirin improves prognosis after ACS. $^{4}$ P2Y12 receptor antagonists act synergistically with aspirin and in the CURE and COMMIT trials dual-anti-platelet therapy (DAPT) with aspirin and clopidogrel was superior to aspirin alone in patients with non-ST elevation (NSTE)-ACS and ST segment elevation myocardial infarction (STEMI). ${ }^{5,6}$ The newer compounds prasugrel and ticagrelor do provide a more intense and better predictable anti-platelet effect than clopidogrel and were associated with a reduction of

ischaemic events in two large trials in patients with ACS. ${ }^{7,8}$ Therefore, these drugs are recommended over clopidogrel in the current European Society of Cardiology (ESC) guidelines. ${ }^{2,3}$ Intravenous or subcutaneous anticoagulation is given in the acute phase during ACS, especially in patients undergoing percutaneous coronary intervention (PCI). ${ }^{9}$ Long-term oral anticoagulation (OAC) with vitamin $\mathrm{K}$ antagonists (VKAs) is more effective than aspirin after acute myocardial infarction in reducing ischaemic events but is associated with more bleeding complications. ${ }^{10,11}$ In addition, this therapy is more cumbersome since regular testing of the international normalized ratio is mandatory, and therefore anticoagulation has not become standard therapy in secondary prevention after ACS. ${ }^{2,3}$ With the advent of PCI with stent implantation as the preferred reperfusion and revascularization therapy, DAPT has been considered @ 2018 Georg Thieme Verlag KG
Stuttgart · New York
DOI https://doi.org/ 10.1055/s-0038-1668133. ISSN 0340-6245. 
mandatory and recommended in current guidelines to prevent stent thrombosis. ${ }^{2,3}$ However, the evidence supporting these recommendations is based on a limited number of trials showing enhanced efficacy and fewer bleeding events with DAPT compared with a combined anti-platelet and anticoagulant strategy. ${ }^{12-14}$ Just recently with the development of novel oral anticoagulants (NOACs), there was a renewed interest in the long-term OAC after ACS. Two large studies have assessed the impact of the addition of a NOAC on standard of care in improving patient outcomes after ACS. ${ }^{15,16}$ This article will discuss the pros and cons of intensified anti-thrombotic therapy with more potent platelet inhibitors versus dual pathway inhibition including oral anticoagulation with low-dose rivaroxaban as secondary prevention therapy after ACS and give recommendations for their use in clinical practice.

\section{Importance of Thrombin Inhibition after ACS}

There is clear evidence that thrombin generation plays a key role in the very early phase in patients with ACSs (-Fig. 1). Therefore, intravenous anticoagulation is recommended in all patients with ACS. ${ }^{9}$ There is continuous thrombin generation even months after ACS, which makes continuous OAC an attractive therapy. ${ }^{17,18}$ The optimal level of OAC during the chronic phase has not been clearly defined yet. However, the results of the PENTUA and OASIS- 5 trial indicate that lower doses might be beneficial, since a low-dose factor Xa (FXa) inhibition with $2.5 \mathrm{mg}$ fondaparinux was as effective as full dose anticoagulation with $2 \times 1 \mathrm{mg} / \mathrm{kg}$ bodyweight enoxaparin, ${ }^{19,20}$ but associated with a lower bleeding rate and a reduced mortality.

\section{Clinical Results of Chronic Thrombin Inhibition after ACS}

There are two large randomized trials available using a direct FXa inhibitor in patients after ACS, the ATLAS-TIMI 52 trial and the APPRAISE- 2 trial. ${ }^{15,16}$ Both trials included patients with ACSs with and without stent implantation. The main difference was the intensity of OAC used. In ATLAS-2, low-dose regimens with $2 \times 2.5 \mathrm{mg}$ and $2 \times 5 \mathrm{mg}$ rivaroxaban were studied, while the APPRAISE- 2 trial compared a full dose anticoagulation with $2 \times 5 \mathrm{mg}$ apixaban with placebo. The APPRAISE- 2 was prematurely stopped because of an increase in bleeding complications with apixaban without any benefit in the primary combined ischaemic endpoint. In contrast, the very low dose of $2 \times 2.5 \mathrm{mg}$ rivaroxaban significantly reduced the combined primary endpoint, as well as cardiovascular (CV) and total mortality in the ATLAS-TIMI 52 trial (- Table 1). As in the APPRAISE-2 trial, an increase in major bleeding complications was observed, while there was no increase in intra-cranial and fatal bleedings. Again, these results support the hypothesis that lower doses of OAC are beneficial for secondary prevention after ACS.

\section{Clinical Benefits of Intensified Platelet Inhibition versus Direct Factor Xa Inhibition after ACS}

The basis for DAPT has been the CURE trial, which showed superiority of clopidogrel over placebo in patients with NSTE-ACS treated with aspirin. The more potent P2Y12 inhibitors prasugrel and ticagrelor further reduced

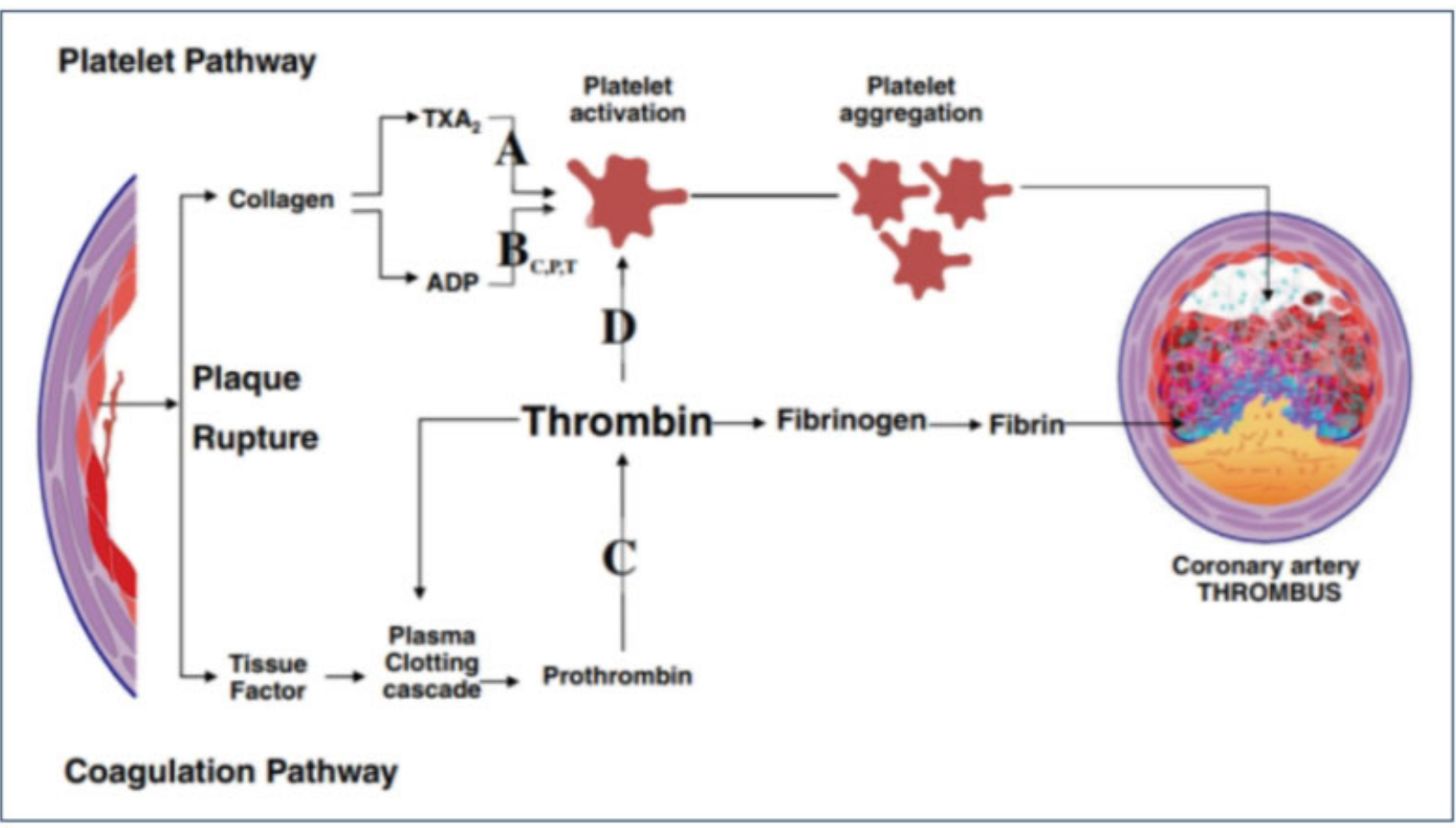

Fig. 1 Contribution of the platelet and coagulation pathways on thrombus formation (Reproduced from Welsh et $\mathrm{al}^{31}$ ). 
Table 1 Features and results of three major trials with intensified anti-thrombotic therapies after acute coronary syndromes

\begin{tabular}{|l|l|l|l|}
\hline & PLATO & TRITON & ATLAS-TIMI 52 \\
\hline Drug & Ticagrelor & Prasugrel & Rivaroxaban \\
\hline Comparator & Clopidogrel & Clopidogrel & $\begin{array}{l}\text { Placebo on top of dual anti- } \\
\text { platelet therapy in } 81 \%\end{array}$ \\
\hline Mean treatment duration & $9.1 \mathrm{mo}$ & $14.5 \mathrm{mo}$ & $13.1 \mathrm{mo}$ \\
\hline CV death/MI/Stroke control group & $11.7 \%$ & $12.1 \%$ & $10.7 \%$ \\
\hline CV death/MI/Stroke control treatment group & $9.8 \%$ & $9.9 \%$ & $9.1 \%$ \\
\hline Relative risk reduction & $16 \%$ & $18 \%$ & $16 \%$ \\
\hline CV death & $5.1 \%$ versus $4.0 \%$ & $2.4 \%$ versus $2.1 \%$ & $4.1 \%$ versus $2.7 \%$ \\
\hline All cause death & $5.9 \%$ versus $4.5 \%$ & $3.2 \%$ versus $3.0 \%$ & $4.5 \%$ versus $2.9 \%$ \\
\hline Non-CABG TIMI major bleeding & 2.2. versus $2.8 \%$ & $1.8 \%$ versus $2.4 \%$ & $1.8 \%$ versus $0.6 \%$ \\
\hline ICH & $0.2 \%$ versus $0.3 \%$ & $0.3 \%$ versus $0.3 \%$ & $0.2 \%$ versus $0.4 \%$ \\
\hline Fatal bleeding & $0.3 \%$ versus $0.3 \%$ & $0.1 \%$ versus $0.4 \%$ & $0.2 \%$ versus $0.1 \%$ \\
\hline Net clinical benefit & $13.9 \%$ versus $12.6 \%$ & $13.9 \%$ versus $12.3 \%$ & $11.3 \%$ versus $10.9 \%$ \\
\hline
\end{tabular}

Abbreviations: CABG, coronary artery bypass grafting; CV, cardiovascular; ICH, intra-cranial haemorrhage; MI, myocardial infarction; TIMI, thrombolysis in myocardial infarction.

ischaemic events compared with clopidogrel in the TRITON and PLATO trials (- Table $\mathbf{1}$ ).

Most likely in part due to differences in patient population and design ( - Table 1 ), CV and total mortality were reduced, while there was no mortality benefit in TRITON.

There are no direct comparisons between an intensified platelet inhibition and a dual pathway inhibition with a combination therapy of anti-platelets plus low-dose antiFXa inhibition on long-term outcome available, and therefore we can only speculate on the comparative value of the two approaches.

One possibility might be an indirect comparison based on the results of the three major randomized trials. Since both ischaemic and bleeding complications are associated with an impaired prognosis after ACS, ${ }^{21,22}$ the ideal antithrombotic therapy should be both effective and safe. Therefore, the term net clinical benefit incorporating both ischaemic and bleeding events has been created and been used to compare anti-thrombotic therapies. However, for the patient and the treating physician these events might not be of the same importance. Thus, combining endpoints to define the clinical value of a given regimen has some limitations. There is general consensus that mortality is the most important endpoint in clinical trials. Ideally, disease-specific mortality should be reduced without an increase in mortality for other causes, resulting in a reduction in all-cause mortality. - Table 1 depicts the results of the three major randomized trials showing a benefit of an intensified anti-thrombotic therapy in patients after ACS.

In summary, both ticagrelor and rivaroxaban were associated with a significant reduction in mortality, which is considered to be the most important endpoint for both patients and physicians.

\section{Aspirin versus Low-Dose Rivaroxaban after ACS}

In a double-blind, multi-centre, randomized trial (GEMINIACS-1), patients with unstable angina, non-ST segment elevation myocardial infarction or STEMI, with positive cardiac biomarkers and either ischaemic electrocardiographic changes or an atherosclerotic culprit lesion identified during angiography, were randomly assigned (1:1) within 10 days after admission for the index ACSs event to either aspirin or rivaroxaban. ${ }^{23}$ Randomization was stratified based on the background P2Y12 inhibitor (clopidogrel or ticagrelor) intended to be used at the time of randomization. Patients received a minimum of 180 days of double-blind treatment with rivaroxaban $2.5 \mathrm{mg}$ twice daily or aspirin $100 \mathrm{mg}$ daily. The primary endpoint was thrombolysis in myocardial infarction (TIMI) clinically significant bleeding not related to coronary artery bypass grafting (CABG; major, minor or requiring medical attention) up to day 390 . Primary analysis was by intention to treat. A total of 3,037 patients with ACSs were randomly assigned; 1,518 to receive aspirin and 1,519 to receive rivaroxaban. A total of 1,704 patients $(56 \%)$ were in the ticagrelor and 1,333 (44\%) in the clopidogrel strata. Median duration of treatment was 291 days (interquartile range, 239354). TIMI non-CABG clinically significant bleeding was similar with rivaroxaban versus aspirin therapy (total 154 patients [5\%]; 80 patients [5\%] of 1,519 vs. 74 patients [5\%] of 1,518 ; hazard ratio [HR], 1.09 [95\% confidence interval, 0.80-1.50]; $p=0.5840$ ). The study was not powered to assess efficacy; however, no differences in the composite ischaemic endpoint of $\mathrm{CV}$ death, non-fatal stroke and myocardial infarction was observed. Despite these results, without any reduction of ischaemic endpoints the combination therapy of a single anti-platelet and low-dose FXa inhibition seems potentially 
attractive. However, further studies are needed to define the optimal duration and intensity of such a combination therapy in patients with ACS.

\section{Low-Dose Rivaroxaban in Patients with Atrial Fibrillation Undergoing PCI}

The PIONEER AF-PCI trial is the first large-scale trial to compare conventional VKA-based combination therapy with a NOAC-based therapy in patients with atrial fibrillation (AF) undergoing $\mathrm{PCI}^{24}$ In this trial, 2,124 participants with $\mathrm{AF}$ undergoing PCI with stenting were randomly assigned to receive, in a 1:1:1 ratio, low-dose rivaroxaban (15 mg once daily with further reduction to $10 \mathrm{mg}$ in patients with creatinine clearance of $30-50 \mathrm{~mL} / \mathrm{min}$ ) plus a P2Y12 inhibitor for 12 months (group 1), very-low-dose rivaroxaban (2.5 mg twice daily) plus DAPT for 1,6 or 12 months (group 2 ) or standard therapy with a dose-adjusted VKA plus DAPT for 1,6 or 12 months (group 3). The primary safety outcome was clinically significant bleeding (a composite of major bleeding or minor bleeding according to TIMI criteria or bleeding requiring medical attention). While there was a significantly lower incidence of bleedings with both rivaroxaban regimens compared with VKA plus DAPT, ischaemic complications, death or stroke rates were similar.

\section{Low-Dose Rivaroxaban in Patients with Chronic Coronary Artery Disease}

In the large COMPASS $^{25}$ trial, 27,395 patients with stable artheroslerotic disease, namely, coronary artery disease in $91 \%$ of patients or peripheral artery disease, were randomized to aspirin alone, $2 \times 5 \mathrm{mg}$ rivaroxaban or combination therapy with aspirin and $2 \times 2.5 \mathrm{mg}$ rivaroxaban. The study was prematurely stopped for superiority of the combination therapy after a mean-follow-up of 23 months. The results are summarized in - Table $\mathbf{2}$. Here, again a reduction

Table 2 Results of the COMPASS trial in the aspirin monotherapy and combination therapy-aspirin $+2 \times 2.5 \mathrm{mg}$ rivaroxaban group

\begin{tabular}{|l|l|l|l|}
\hline & Aspirin & $\begin{array}{l}\text { Aspirin }+2 \\
\times 2.5 \mathrm{mg} \\
\text { rivaroxaban }\end{array}$ & $p$-Value \\
\hline Patients $(n)$ & 9,126 & 9,152 & \\
\hline $\begin{array}{l}\text { Combined primary } \\
\text { endpoint }\end{array}$ & $5.4 \%$ & $4.1 \%$ & $<0.001$ \\
\hline CV mortality & $2.2 \%$ & $1.7 \%$ & 0.02 \\
\hline Non-fatal MI & $2.2 \%$ & $1.9 \%$ & 0.14 \\
\hline Non-fatal stroke & $1.6 \%$ & $0.9 \%$ & $<0.001$ \\
\hline All-cause mortality & $4.1 \%$ & $3.4 \%$ & 0.01 \\
\hline Major bleeding & $1.9 \%$ & $3.1 \%$ & $<0.001$ \\
\hline Fatal bleeding & $0.1 \%$ & $0.2 \%$ & 0.32 \\
\hline Intra-cranial bleeding & $0.3 \%$ & $0.3 \%$ & 0.60 \\
\hline
\end{tabular}

Abbreviations: CV, cardiovascular; MI, myocardial infarction. in CV and total mortality could be observed with rivaroxaban $2 \times 2.5 \mathrm{mg}$ with an increase in major bleeding complications but not in fatal bleedings or intra-cranial haemorrhage. Of special interest were the results in the patients with peripheral artery disease. In these patients, not only major adverse cardiovascular events (MACEs) were reduced but also amputations and lower limb events. While there was no significant benefit in the $2 \times 5 \mathrm{mg}$ rivaroxaban group, the curves continued to diverge in favour of rivaroxaban versus aspirin, indicating that OAC with FXa inhibition is at least as effective as platelet inhibition for secondary prevention in patients with coronary artery disease and/or peripheral artery disease.

The results of COMPASS have to be seen in the light of the PEGASUS $^{26}$ trial, which evaluated longer term use of ticagrelor $>12$ months after an ACS. As in COMPASS, the primary combined endpoint of $\mathrm{CV}$ death, myocardial infarction and stroke was reduced with ticagrelor versus placebo. Therefore, physicians currently have two options for an intensified anti-thrombotic therapy in high-risk patients with coronary artery disease $<12$ months after ACS.

\section{Hurdles against the Use of Rivaroxaban after ACS}

Patients with coronary stent placement and an indication for OAC for diverse indications, like AF as well as mechanical heart valves, are in need of distinct medical regimes. This treatment with full dose of OAC together with DAPT is termed "triple therapy." This triple therapy is known to be associated with an increased risk of bleeding events as shown by a recent meta-analysis. ${ }^{27}$ As mentioned before, bleeding events are further known to have a negative impact on outcome, since they are associated with high risk of $\mathrm{CV}$ events including recurrent myocardial infarction, acute stent thrombosis and death. Therefore, physicians try to avoid the triple therapy whenever possible. This is aggravated by the fact, that indeed physicians rate bleeding events as especially important, potentially because those could be directly or indirectly connected to individual treatment decisions of the distinct physician. This fear may be based on the very foundation of medical therapies in view of the "Nihil Nocere" principle, which targets to prevent harm before achieving any possible benefit.

Generally, this might explain that uptake of a medical strategy that applies OAC, even at very low dosages, combined with DAPT was slow so far, even when data showing beneficial effects are available. Interestingly, uptake of novel P2Y12 receptor inhibitors in patients with ACS was faster, ${ }^{28}$ although these agents also increase bleeding events as more potent inhibitors of platelet function compared with clopidogrel. This disparity in physician's treatment choice between increasing anti-platelet therapy compared with targeting another molecular mechanism in view of the dual pathway approach needs to be further elucidated.

To overcome this hurdle in the application of dual pathway strategy including low-dose OAC with DAPT, physicians need to consider the net clinical benefit of this strategy. More 
studies should demonstrate the overall safety of this aspect and clearly address the risk-benefit trade-offs.

Another important aspect of treatment preference might include different complexity of drug regimens. This is important for drug persistence, an important part of efficacy of any medical strategy, especially in the older CV disease patients, where total pill count is high. The number of drugs is one easy predicator of drug persistence after a distinct treatment period. ${ }^{29}$ Therefore, adding tablets to the daily regimen is not the preferred choice of many physicians. This is especially important, since lowering the pill count is one major goal in addressing other CV diseases like hypertension or lipid-lowering treatments, where combination therapies are becoming more popular choices. Therefore, replacing clopidogrel with another P2Y12 receptor inhibitor might be preferred by many physicians compared with adding a twice-daily regimen.

This might be further complicated by the fact that knowledge about the availability of low-dose rivaroxaban is still limited, especially in prescribing general practitioners. This bears the practical risk of miss-prescribing other NOACs instead of rivaroxaban $2.5 \mathrm{mg}$ twice daily, e.g., apixaban, given that the $2.5 \mathrm{mg}$ twice daily dose is very well known for stroke prevention. This miss-prescription might translate into higher bleeding risk, given this would be a full dose OAC given mistakenly on top of DAPT.

Finally, the current guidelines of the $\operatorname{ESC}^{2,3}$ do not support the use of rivaroxaban, with only a class IIb recommendation, stating: "in low-bleeding risk patients who receive aspirin and clopidogrel low-dose rivaroxaban (2.5 mg twice daily) may be considered, while DAPT with prasugrel ticagrelor is a class I indication."

\section{Patient Selection for the Use of Rivaroxaban after ACS}

Given the discussed evidence of the dual pathway approach and weighing it against the hurdles of adding even a lowdose OAC to DAPT therapy, it is of utmost importance to discuss the characteristics of suitable patients for this treatment regimen. While data would support treating all patients stabilized after an ACS with this therapy, some patient profiles might be especially compelling for the dual pathway approach, although specific study data to choose distinct patients population do not exist yet (-Table 3 ).

First, patients with the rare, but potentially deadly event of an acute stent thrombosis are in need of intensified treatment. While modern P2Y12 receptor inhibitors are known to decrease the number of these events, the same is true for adding low-dose rivaroxaban to acetylsalicylic acid and clopidogrel. It would be intriguing to add low-dose rivaroxaban in patients suffering from acute stent thrombosis despite treatment with a modern P2Y12 receptor inhibitor. In this scenario, physicians need to replace the novel P2Y12 receptor inhibitor with clopidogrel, but distinct targeting of the thrombin pathway might be warranted to inhibit exacerbation of platelet aggregation. Importantly, early as well as late presenters with stent thrombosis need
Table 3 Patient populations potentially especially suitable for dual pathway inhibition after an acute coronary syndrome

\begin{tabular}{|l|}
\hline Stent thrombosis under DAPT \\
\hline Thrombotic occlusion of a saphenous vein graft \\
\hline High thrombus burden in the acute phase \\
\hline Flow-limiting coronary aneurysms \\
\hline $\begin{array}{l}\text { Coronary plaque erosion without high grade stenosis } \\
\text { treated medically }\end{array}$ \\
\hline $\begin{array}{l}\text { Atrial fibrillation and high risk for stent thrombosis } \\
\text { (multiple stents, long stents, bifurcation lesions) }\end{array}$ \\
\hline $\begin{array}{l}\text { Accompanying symptomatic peripheral artery disease or } \\
\text { prior peripheral re-vascularization }\end{array}$ \\
\hline
\end{tabular}

Abbreviation: DAPT, dual-anti-platelet therapy.

to be evaluated for stent malapposition using modern coronary imaging technology in addition to potent anti-thrombotic treatment.

Second, patients with failure of the saphenous vein grafts are a sub-population in need of special medical care. Thrombotic material is present in many of the failing saphenous veins making targeting the anticoagulation pathway theoretically attractive, especially after stent placement in those vein grafts. This is further strengthened by the fact that $\mathrm{PCI}$ in the saphenous vein grafts is associated with higher rates of thrombotic complications like no-reflow syndrome.

Third, and in this view, patients presenting with excessive native coronary thrombus formation as well as slow coronary flow or even no-reflow syndrome after ACS-PCI are often treated with potent anti-platelet drugs, like glycoprotein IIa/ IIIb receptor inhibitors on top of DAPT during the PCI or the adjunctive hours. While starting the dual pathway approach should only be considered after stabilization of the patients, e.g., days after the index event, this scenario where large thrombus burden occurred leading to macro-vascular and micro-vascular obstruction might represent a sub-set of patients in need of potent anti-thrombotic strategies exhibited by rivaroxaban.

Fourth, patients presenting with coronary anomalies like flow-limiting aneurysms are difficult to treat and known to have increased risk of coronary events. Many physicians add full dose OAC to DAPT in these patients to target the coagulation systems potently. While no controlled data are available for this treatment, it might be preferred to add lowdose rivaroxaban to aspirin and clopidogrel given the safety of this approach compared with DAPT and full dose OAC as discussed above. Nevertheless, there is no evidence-based data guiding this strategy, but individual treatment decision might include the dual pathway strategy.

Fifth, an emerging pathology underling ACS is plaque erosion visualized mostly by optical coherence tomography. Plaque erosion often cause substantial thrombus formation attached to the endothelial lesion limiting blood flow. No controlled data are available for these patients, suggested to be the trigger of ACS in one-fourth of patients. Interestingly, it was suggested in a small study, that medical management 
alone without coronary stenting might be sufficient to reduce thrombus mass associated with these lesions and this was associated with high safety. Adding a low-dose OAC in patients with high thrombus burden seems intriguing in view of the underlying pathophysiology, but warrants further research.

Sixth, a very compelling application of the dual pathway approach is in patients after coronary stenting and underlying AF in need of pharmacological stroke prevention. In these patients, OAC as well as anti-platelet strategy is urgently needed to reduce ischaemic complications in view of the coronary stents as well as for the prevention of embolic stroke. The above discussed Pioneer AF-PCI study represents the best dataset yet for treating these patients. One could argue to choose a DAPT treatment together with low-dose rivaroxaban regimen especially in patients with complex coronary disease, high stent length or coronary lesions that are associated with adverse events like bifurcation lesion treated with a two-stent strategy. Again, no data exist to persuasively decipher where this treatment strategy might be especially advantageous, but many physicians would argue in favour of DAPT plus low-dose rivaroxaban in these patient sub-populations.

Seventh, in the light of the results of the COMPASS study, ${ }^{30}$ patients with ACS and peripheral artery disease, a population with a high risk for adverse events, might be an attractive group, which would have benefit of this dual pathway inhibition both for MACE and limb events.

Unfortunately, there are little data to back up the efficacy of low-dose FXa inhibition in these selected patient cases. ${ }^{31}$ While making sense form a pathophysiological perspective, the clinical trials do not have large sub-groups with the former mentioned patients. Best data might exist for patients with stent thrombosis. In the ATLAS-TIMI 51 trial, rivaroxaban reduced the incidence of primary stent thrombosis from 1.9 to $1.5 \%$ (HR, 0.65 [0.46-0.93]) significantly. Coherently, these patient vignettes are a model of thought where this novel therapy might be applied.

\section{Conclusion}

New approaches in anti-thrombotic therapies, such as DAPT, will usually need some time get a place in clinical practice. This is due to several factors such as complexity of the new approach, familiarity and confidence with the established therapy. As mentioned above, the fear of bleeding is another major concern of physicians not introducing a new, more potent anti-thrombotic regimen.

However, these considerations should not prohibit physicians to apply effective and possibly life-saving therapies to their patients. To gain some experience, it might be worthwhile to start using therapies in selected patient populations; for low-dose rivaroxaban, these examples are described above. The positive results of the COMPASS trial, which were recently published, will further increase interest in combination therapy of low-dose FXa inhibition and platelet inhibition.
Conflict of Interest

U.Z.: Research funding from Eli Lilly, Germany, Daiichi Sankyo, Germany, Sanofi, Germany; Speakers honoraria: Bayer, Astra Zeneca, Daiichi Sankyo, Eli Lilly and Sanofi. B.S.: None. D.W.: Speakers honoraria: Bayer, Astra Zeneca and Daiichi Sankyo.

\section{References}

1 Lippi G, Franchini M, Targher G. Arterial thrombus formation in cardiovascular disease. Nat Rev Cardiol 2011;8(09):502-512

2 Roffi M, Patrono C, Collet J-P, et al; ESC Scientific Document Group. 2015 ESC guidelines for the management of acute coronary syndromes in patients presenting without persistent ST-segment elevation: Task Force for the Management of Acute Coronary Syndromes in Patients Presenting without Persistent ST-Segment Elevation of the European Society of Cardiology (ESC). Eur Heart J 2016;37(03):267-315

3 Ibanez B, James S, Agewall S, et al; ESC Scientific Document Group. 2017 ESC guidelines for the management of acute myocardial infarction in patients presenting with ST-segment elevation: the Task Force for the management of acute myocardial infarction in patients presenting with ST-segment elevation of the European Society of Cardiology (ESC). Eur Heart J 2018;39(02):119-177

4 Baigent C, Blackwell L, Collins R, et al; Antithrombotic Trialists' (ATT) Collaboration. Aspirin in the primary and secondary prevention of vascular disease: collaborative meta-analysis of individual participant data from randomised trials. Lancet 2009; 373(9678):1849-1860

5 Yusuf S, Zhao F, Mehta SR, Chrolavicius S, Tognoni G, Fox KK; Clopidogrel in Unstable Angina to Prevent Recurrent Events Trial Investigators. Effects of clopidogrel in addition to aspirin in patients with acute coronary syndromes without ST-segment elevation. N Engl J Med 2001;345(07):494-502

6 Chen ZM, Jiang LX, Chen YP, et al; COMMIT (ClOpidogrel and Metoprolol in Myocardial Infarction Trial) collaborative group. Addition of clopidogrel to aspirin in 45,852 patients with acute myocardial infarction: randomised placebo-controlled trial. Lancet 2005;366(9497):1607-1621

7 Wiviott SD, Braunwald E, McCabe $\mathrm{CH}$, et al; TRITON-TIMI 38 Investigators. Prasugrel versus clopidogrel in patients with acute coronary syndromes. N Engl J Med 2007;357(20):2001-2015

8 Wallentin L, Becker RC, Budaj A, et al; PLATO Investigators. Ticagrelor versus clopidogrel in patients with acute coronary syndromes. N Engl J Med 2009;361(11):1045-1057

9 Zeymer U, Rao SV, Montalescot G. Anticoagulation in coronary intervention. Eur Heart J 2016;37(45):3376-3385

10 van Es RF, Jonker JJ, Verheugt FW, Deckers JW, Grobbee DE; Antithrombotics in the Secondary Preventionof Events in Coronary Thrombosis-2 (ASPECT-2) Research Group. Aspirin and coumadin after acute coronary syndromes (the ASPECT-2 study): a randomised controlled trial. Lancet 2002;360(9327):109-113

11 Hurlen M, Abdelnoor M, Smith P, Erikssen J, Arnesen H. Warfarin, aspirin, or both after myocardial infarction. N Engl J Med 2002; 347(13):969-974

12 Schömig A, Neumann F-J, Kastrati A, et al. A randomized comparison of antiplatelet and anticoagulant therapy after the placement of coronary-artery stents. N Engl J Med 1996;334(17):1084-1089

13 Bertrand ME, Legrand V, Boland J, et al. Randomized multicenter comparison of conventional anticoagulation versus antiplatelet therapy in unplanned and elective coronary stenting. The full anticoagulation versus aspirin and ticlopidine (fantastic) study. Circulation 1998;98(16):1597-1603

14 Leon MB, Baim DS, Popma JJ, et al; Stent Anticoagulation Restenosis Study Investigators. A clinical trial comparing three 
antithrombotic-drug regimens after coronary-artery stenting. N Engl J Med 1998;339(23):1665-1671

15 Hess CN, James S, Lopes RD, et al. Apixaban plus mono versus dual antiplatelet therapy in acute coronary syndromes: insights from the APPRAISE-2 Trial. J Am Coll Cardiol 2015;66(07):777-787

16 Mega JL, Braunwald E, Wiviott SD, et al; ATLAS ACS 2-TIMI 51 Investigators. Rivaroxaban in patients with a recent acute coronary syndrome. N Engl J Med 2012;366(01):9-19

17 Ardissino D, Merlini PA, Bauer KA, et al. Coagulation activation and long-term outcome in acute coronary syndromes. Blood 2003;102(08):2731-2735

18 Merlini PA, Bauer KA, Oltrona L, et al. Persistent activation of coagulation mechanism in unstable angina and myocardial infarction. Circulation 1994;90(01):61-68

19 Simoons ML, Bobbink IWG, Boland J, et al; PENTUA Investigators. A dose-finding study of fondaparinux in patients with non-STsegment elevation acute coronary syndromes: the Pentasaccharide in Unstable Angina (PENTUA) Study. J Am Coll Cardiol 2004 43(12):2183-2190

20 Yusuf S, Mehta SR, Chrolavicius S, et al; Fifth Organization to Assess Strategies in Acute Ischemic Syndromes Investigators. Comparison of fondaparinux and enoxaparin in acute coronary syndromes. N Engl J Med 2006;354(14):1464-1476

21 Eikelboom JW, Mehta SR, Anand SS, Xie C, Fox KAA, Yusuf S. Adverse impact of bleeding on prognosis in patients with acute coronary syndromes. Circulation 2006;114(08):774-782

22 Jokhadar M, Jacobsen SJ, Reeder GS, Weston SA, Roger VL. Sudden death and recurrent ischemic events after myocardial infarction in the community. Am J Epidemiol 2004;159(11):1040-1046

23 Ohman EM, Roe MT, Steg PG, et al. Clinically significant bleeding with low-dose rivaroxaban versus aspirin, in addition to P2Y12 inhibition, in acute coronary syndromes (GEMINI-ACS-1): a double-blind, multicentre, randomised trial. Lancet 2017; 389(10081):1799-1808

24 Gibson CM, Mehran R, Bode C, et al. Prevention of bleeding in patients with atrial fibrillation undergoing PCI. N Engl J Med 2016;375(25):2423-2434

25 Eikelboom JW, Connolly SJ, Bosch J, et al; COMPASS Investigators. Rivaroxaban with or without aspirin in stable cardiovascular disease. N Engl J Med 2017;377(14):1319-1330

26 Bonaca MP, Bhatt DL, Cohen M, et al; PEGASUS-TIMI 54 Steering Committee and Investigators. Long-term use of ticagrelor in patients with prior myocardial infarction. N Engl J Med 2015; 372(19):1791-1800

27 Andrade JG, Deyell MW, Khoo C, Lee M, Humphries K, Cairns JA. Risk of bleeding on triple antithrombotic therapy after percutaneous coronary intervention/stenting: a systematic review and meta-analysis. Can J Cardiol 2013;29(02):204-212

28 Angerås $\mathrm{O}$, Hasvold $\mathrm{P}$, Thuresson $\mathrm{M}$, Deleskog $\mathrm{A}$, ÖBraun $\mathrm{O}$. Treatment pattern of contemporary dual antiplatelet therapies after acute coronary syndrome: a Swedish Nationwide Population-Based Cohort study. Scand Cardiovasc J 2016;50(02):99-107

29 Thayer S, Arondekar B, Harley C, Darkow TE. Adherence to a fixeddose combination of rosiglitazone/glimepiride in subjects switching from monotherapy or dual therapy with a thiazolidinedione and/or a sulfonylurea. Ann Pharmacother 2010;44(05):791-799

30 Anand SS, Bosch J, Eikelboom JW, et al; COMPASS Investigators. Rivaroxaban with or without aspirin in patients with stable peripheral or carotid artery disease: an international, randomised, double-blind, placebo-controlled trial. Lancet 2017: S0140-6736(17)32409-1

31 Welsh RC, Roe MT, Steg PG, et al. A critical reappraisal of aspirin for secondary prevention in patients with ischemic heart disease. Am Heart J 2016;181:92-100 